\title{
Clinical impact and network of determinants of tumour necrosis in colorectal cancer
}

\author{
Sara A Väyrynen ${ }^{1,2,5}$, Juha P Väyrynen ${ }^{1,2,5}$, Kai Klintrup ${ }^{3,4}$, Jyrki Mäkelä ${ }^{3,4}$, Tuomo J Karttunen ${ }^{1,2}$, \\ Anne Tuomisto ${ }^{1,2}$ and Markus J Mäkinen ${ }^{\star, 1,2}$ \\ ${ }^{1}$ Department of Pathology, Cancer and Translational Medicine Research Unit, University of Oulu, POB 5000, Oulu 90014, Finland; \\ ${ }^{2}$ Department of Pathology, Oulu University Hospital and Medical Research Center Oulu, POB 21, Oulu 90029, Finland; \\ ${ }^{3}$ Department of Surgery, Oulu University Hospital and Medical Research Center Oulu, POB 21, Oulu 90029, Finland and \\ ${ }^{4}$ Department of Surgery, Research Unit of Surgery, Anesthesia and Intensive Care, University of Oulu, POB 5000, Oulu 90014, \\ Finland
}

Background: The disease outcome in colorectal cancer (CRC) can vary in a wide range within the same tumour stage. The aim of this study was to clarify the prognostic value and the determinants of tumour necrosis in CRC.

\begin{abstract}
Methods: The areal proportion (\%) of tumour tissue showing coagulative necrosis was evaluated in a cohort of 147 CRC patients and correlated with basic clinicopathological characteristics, microvascular density (MVD), cell proliferation rate, KRAS and BRAF mutations, and survival. To validate the prognostic significance of tumour necrosis, an independent cohort of 418 CRC patients was analysed.

Results: Tumour necrosis positively correlated with tumour stage ( $P=8.5 \mathrm{E}-4)$ —especially with T class (4.0E - 6)—and inversely correlated with serrated histology $(P=0.014)$, but did not significantly associate with cell proliferation rate, MVD, and KRAS or BRAF mutation. Abundant (10\% or more) tumour necrosis associated with worse disease-free survival independent of stage and other biological or clinicopathological characteristics in both cohorts, and the adverse effect was directly related to its extent. High CD105 MVD was also a stage independent marker for worse disease-free survival.
\end{abstract}

Conclusions: Tumour necrosis percentage is a relevant histomorphological prognostic indicator in CRC. More studies are needed to disclose the mechanisms of tumour necrosis.

Colorectal cancer (CRC) is one of the most common cancers and causes of cancer deaths worldwide (Center et al, 2009). Currently, the prognostic classification of CRC is mostly based on the TNM staging (Hamilton et al, 2010). However, the behaviour of the tumour can vary widely within the same tumour stage (Puppa et al, 2010), and new tools for the evaluation of prognosis are needed.

Tumour necrosis is a feature often presented in human solid tumours. Extensive necrosis has been reported to associate with worse survival in CRC (Mulcahy et al, 1997; Gao et al, 2005; Pollheimer et al, 2010; Richards et al, 2012), as well as in other solid tumours including lung cancer (Swinson et al, 2002) and renal cancer (Frank et al, 2002). However, predetermined cutoff scores or categorisations in necrosis evaluation may have affected the results of the previous studies (Mulcahy et al, 1997; Gao et al, 2005; Pollheimer et al, 2010; Richards et al, 2012), while the evaluation of necrosis percentage as a continuous variable would enable more sophisticated statistical methods such as ROC analysis and linear regression (Zlobec et al, 2007).

It is not clear why some tumours contain abundant necrosis. An obvious explanation would be that tumour necrosis reflects intratumoral hypoxia and results from rapid tumour cell growth, exceeding the vascular supply of oxygen. This hypothesis is supported by the finding that high proliferation rate is associated with tumour necrosis in renal cell carcinoma (Pichler et al, 2012)

\footnotetext{
${ }^{*}$ Correspondence: Professor MJ Mäkinen; E-mail: markus.makinen@oulu.fi

${ }^{5}$ These authors contributed equally to this work.
} 
and nodular melanoma (Bachmann et al, 2008). However, also controversial results exists (Tollefson et al, 2007; Dutta et al, 2012). In $\mathrm{CRC}$, the association between tumour necrosis and proliferation is unclear. Moreover, it has not been established, whether tumour necrosis in CRC associates with low tumour microvascular density (MVD), possibly reflecting the insufficient vascular supply of the tumour.

Mitogen-activated protein kinase-extracellular signal-regulated kinase (MAPK-ERK) pathway mediates fundamental cellular processes such as cell proliferation, differentiation, growth and apoptosis as a response to extracellular signals (Mäkinen, 2007), and oncogenic mutations in KRAS and BRAF, participants of the MAPK-ERK pathway, occur in about 50\% of CRC (Stefanius et al, 2011). Especially, colorectal serrated adenocarcinomas (SACs) CRCs originating from the serrated polyps and representing at least one of tenth of all CRCs (Mäkinen, 2007)-frequently ( $>75 \%$ ) possess BRAF or KRAS mutation (Stefanius et al, 2011). A few studies have indicated tumour necrosis being less frequent and less extensive in SAC as compared with conventional carcinoma (CC) (Tuppurainen et al, 2005), leading us to hypothesise that the activation of MAPK-ERK pathway, through oncogenic mutations of KRAS or BRAF, may induce mechanisms that prevent tumour necrosis in CRC.

The aim of this study was to clarify the determinants and the clinical impact of tumour necrosis in CRC. We evaluated the areal percentage of microscopic coagulative necrosis within tumours in a cohort of 147 CRC patients and analysed its association with clinicopathological characteristics, including MVD, proliferation rate, KRAS and BRAF mutations, and survival. To validate the prognostic significance of tumour necrosis, an independent cohort of 418 CRC patients was analysed.

\section{MATERIALS AND METHODS}

Patients. This study was based on 147 (99.3\%) of an earlier described prospectively recruited group of 148 CRC patients operated in Oulu University Hospital in 2006-2010 (Supplementary Table S1; Kantola et al, 2012). One $(0.7 \%)$ case was excluded due to insufficiency of sample material. A total of 31 (21.1\%) patients in the study cohort received preoperative radiotherapy or chemoradiotherapy (RT/CRT), which was given to rectal cancer patients with a T3 or T4 tumour. The follow-up data were collected from the case records and from Statistics Finland (Kantola et al, 2014; Väyrynen et al, 2016). Study end points were disease-free survival (DFS), cancer-specific survival (CSS) and overall survival (OS). For validation of the prognostic significance of tumour necrosis, an earlier described group of 418 patients (Validation cohort; reviewed by two experienced gastrointestinal pathologists, MJM and TJK) was included, who underwent an operation for CRC in Oulu University hospital between the years 1986-1996 (Supplementary Table S1; Väyrynen et al, 2014). The 60-month survival data for 352 patients $(84.2 \%)$ was provided by Finnish Cancer Registry. The study design was approved by the Ethical Committee of Oulu University Hospital (58/2005, 184/2009). The reporting recommendations for tumour marker prognostic studies (REMARK) were taken into account in the study design (McShane et al, 2005).

Histopathological analysis. TNM6 (Sobin and Wittekind, 2002) was used in staging and World Health Organization (WHO) criteria in grading the differentiation (Hamilton et al, 2010). The SACs were detected by the WHO 2010 criteria as described earlier (Hamilton et al, 2010; Sajanti et al, 2014), including saw-toothed epithelial serrations, clear or eosinophilic cytoplasm, vesicular nuclei with distinct nucleoli, well-preserved polarity, and abundant mucin production. Tumour growth pattern at the advancing tumour border was classified using the earlier described criteria (Jass et al, 1996), briefly diffuse, irregular clusters or small glands or cords of cells infiltrating to surrounding tissue $v s$ expanding, well-circumscribed margins. Lymphatic invasion was defined as tumour cells present in vessels with an endothelial lining but lacking a muscular wall, and blood vessel invasion was evaluated positive if there were tumour cells in vessels with a thick muscular wall or in vessels containing red blood cells. The areal percentage of tumour necrosis was visually estimated by inspecting manually all available tumour slides. The method was otherwise analogous with two previous studies (Pollheimer et al, 2010; Richards et al, 2012), but no predetermined cutoff scores were utilised in this study. Tumour necrosis in haematoxylin and eosin (H\&E)-stained sections was specified as an area with increased eosinophilia and nuclear shrinkage, fragmentation and disappearance, with shadows of tumour cells visible to variable extent (Figure 1). Neutrophilic inflammatory infiltrate at the boundaries of an area was considered to support the classification of that area as necrotic but was not required by definition. Intraluminal necrosis fulfilled the criteria and was included in the evaluation of tumour necrosis percentage. All the histological analyses were performed blinded to the clinical data.

Computer-assisted measurement of tumour necrosis percentage. To evaluate the accuracy of the visual assessment of tumour necrosis, one H\&E stained section per case of 50 randomly selected CRC cases was scanned using the Aperio AT2 image-capturing device (Leica Biosystems, Wetzlar, Germany). Imagescope software 11.2 (Aperio Technologies, Vista, CA, USA) was used to view the scanned images, and one image was captured per each case at $\times 8$ magnification. Two researchers independently performed visual necrosis percentage estimations for each image and after this, tumour necrosis area was demarcated by hand and its proportion was calculated with ImageJ (US National Institutes of Health, Bethesda, MD, USA), utilising the analyse particles tool.

Tissue microarray. Tissue microarray (TMA) was used in immunohistochemical analyses. The array had been constructed as earlier described (Väyrynen et al, 2013). Briefly, depending on the size of the tumour, 1-4 (median 3 ) cores of $3.0 \mathrm{~mm}$ diameter were manually sampled from each tumour yielding an overall tumour area of $7.1-28.3 \mathrm{~mm}^{2}$. Of these cores, 1-3 (median 2) were taken from the invasive front of the tumour containing the point of deepest invasion and the rest from intratumoural locations.

Immunohistochemistry. Immunohistochemistry was conducted on $3.5 \mu \mathrm{m}$ sections cut from the TMA blocks. The antibodies and the protocols used are summarised in Supplementary Table S2. Mismatch repair (MMR) enzyme screening status for MLH1 and MSH2 was evaluated as described earlier (Väyrynen et al, 2012).

BRAF and KRAS mutation analysis. BRAF and KRAS mutation analyses for CRC cases have been described earlier (Sajanti et al, 2014).

Estimation of microvascular density and proliferation rate. The immunohistochemically stained sections were scanned using Aperio AT2 image-capturing device (Leica Biosystems) at a $\times 20$ magnification. Imagescope software 11.2 (Aperio Technologies) was used to view the scanned images, and one to four images for each case were captured at a $\times 10$ magnification from the areas representing the highest number of CD31, CD105 and von Willebrand factor (vWF, factor-VIII-related antigen) microvessels per area. The numbers of microvessels, recognised by the criteria established earlier (Weidner et al, 1991) were counted from each picture, and the final MVD for each case was defined as the mean value among the individual hot spots. The proliferation rate for each case was assessed with Ki-67 immunohistochemistry and was determined as the mean proportion of positive tumour cell nuclei 

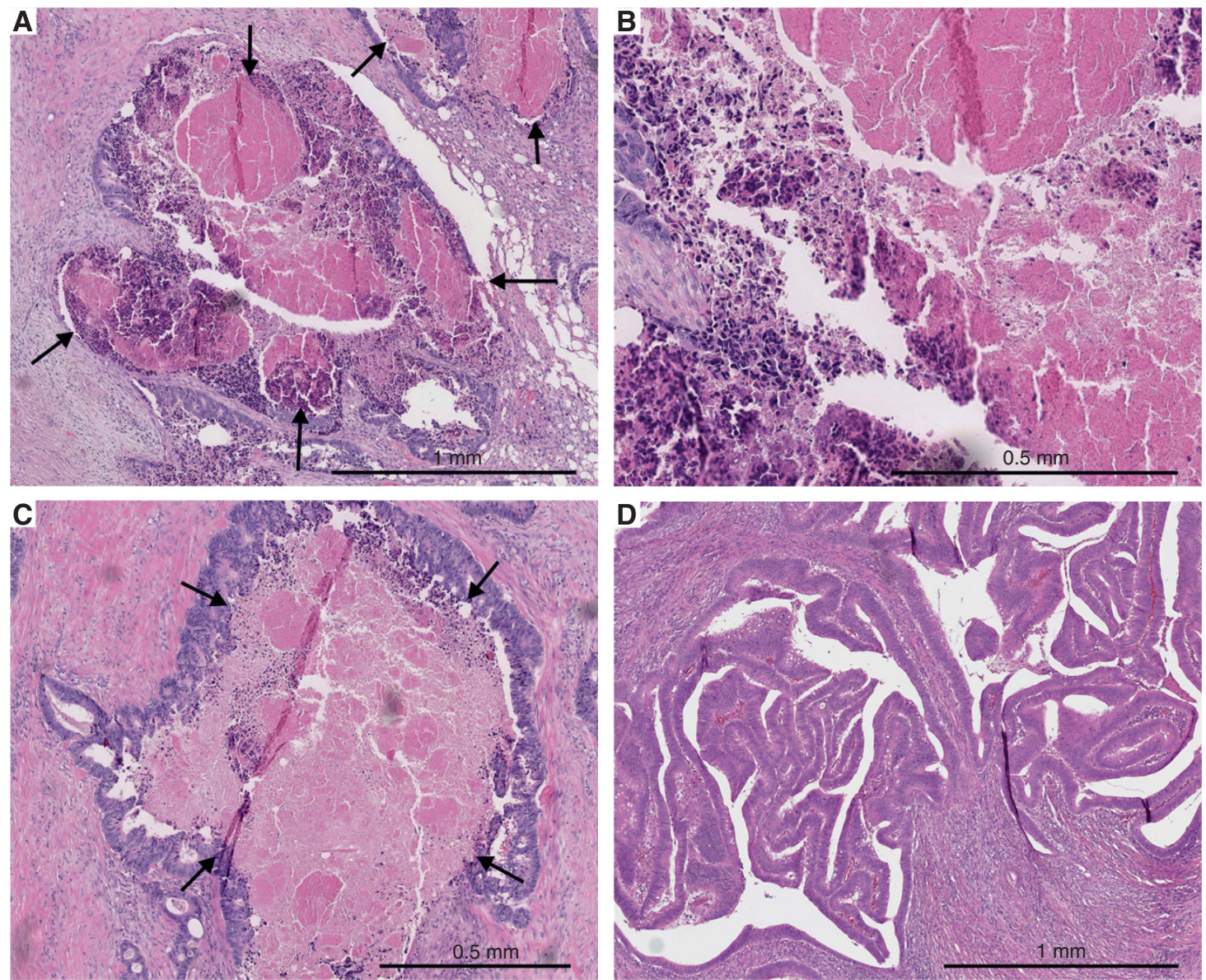

Figure 1. Tumour necrosis in colorectal cancer. (A) Low-power haematoxylin and eosin stained section showing abundant tumour necrosis (arrows). (B) Close-up view from the same case displaying fragments of tumour cells and inflammatory infiltrate that are frequently found in the edges of the necrotic areas. (C) Haematoxylin and eosin stained section of another case showing abundant tumour necrosis (arrows). Intraluminal necrosis such as in this example was also included in the analysis. (D) Haematoxylin and eosin stained section showing scarce tumour necrosis.

in the TMA sections. For Ki-67 and CD31, different tumour areas (invasive front and tumour center) were analysed separately in 30 cases. The values at the invasive front and tumour center had high correlations with each other (Ki-67: Pearson's $r=0.886$; CD31: $r=0.879)$. Therefore, and to adhere to previously described criteria (Des Guetz et al, 2006; Roxburgh et al, 2013), one mean value was recorded for each case. All the immunohistochemical analyses were performed blinded to the clinical data.

Statistical analyses. Normally distributed continuous variables are presented as mean (s.d.) while other continuous variables are presented as median (IQR). The statistical analyses were carried out using IBM SPSS Statistics 22.0 (IBM, Chicago, IL, USA). To test the statistical significance between two categorical variables, $\chi^{2}$ or Fisher's exact test was used. The statistical significance of the associations of tumour necrosis percentage with clinicopathological characteristics was analysed by Mann-Whitney $U$-test (two classes) or Kruskal-Wallis test (three or more classes). Pearson's coefficients $(r)$ were applied in examining correlations between continuous variables, and logarithmic transformation was applied to the variables with positive skewness to accomplish a more normal distribution. Cytoscape, an open source software platform for visualising complex networks, was used in creating a $2 \mathrm{D}$ visualisation of the relationships between tumour necrosis and clinicopathological variables with the Prefuse force directed algorithm weighted by the statistical significances of the associations between individual variables (Shannon et al, 2003). Receiver operating characteristics (ROC) analysis was used to define an optimal cutoff scores, with the shortest distance to the coordinate
$(0,1)$, for tumour necrosis and immunohistochemical markers (CD31, vWF, CD105 and Ki-67) in discriminating survivors from nonsurvivors (Zlobec et al, 2007). DFS, CSS and OS were measured and univariate survival analysis was performed according to the Kaplan-Meier method. Cox's proportional hazards regression model was conducted to analyse the independent prognostic significance of tumour necrosis. Pearson's $r$ and $\kappa$ coefficients were used to evaluate intra- and interobserver agreement. A two-tailed $P$-value $<0.05$ was considered statistically significant.

\section{RESULTS}

Reproducibility and accuracy of the evaluation of tumour necrosis. Two researchers evaluated the tumour necrosis percentage visually and with a computer-assisted method in images of a randomly selected group of $50 \mathrm{CRC}$ patients to estimate the accuracy of the visual assessments of tumour necrosis. The agreement was measured for necrosis as a continuous variable (Pearson's $r$ ) and as a categorical variable with a cutoff point of $10 \%$ ( $\kappa$ score). The correlations between the visual and computerassisted evaluations were excellent (Observer 1: $r=0.930$, $\kappa=0.776$; Observer 2: $r=0.879, \kappa=0.776)$, indicating that visual estimation of tumour necrosis percentage was accurate. Visual estimations of different observers were also highly correlated $(r=0.823, \quad \kappa=0.745)$, indicating an excellent interobserver agreement. 


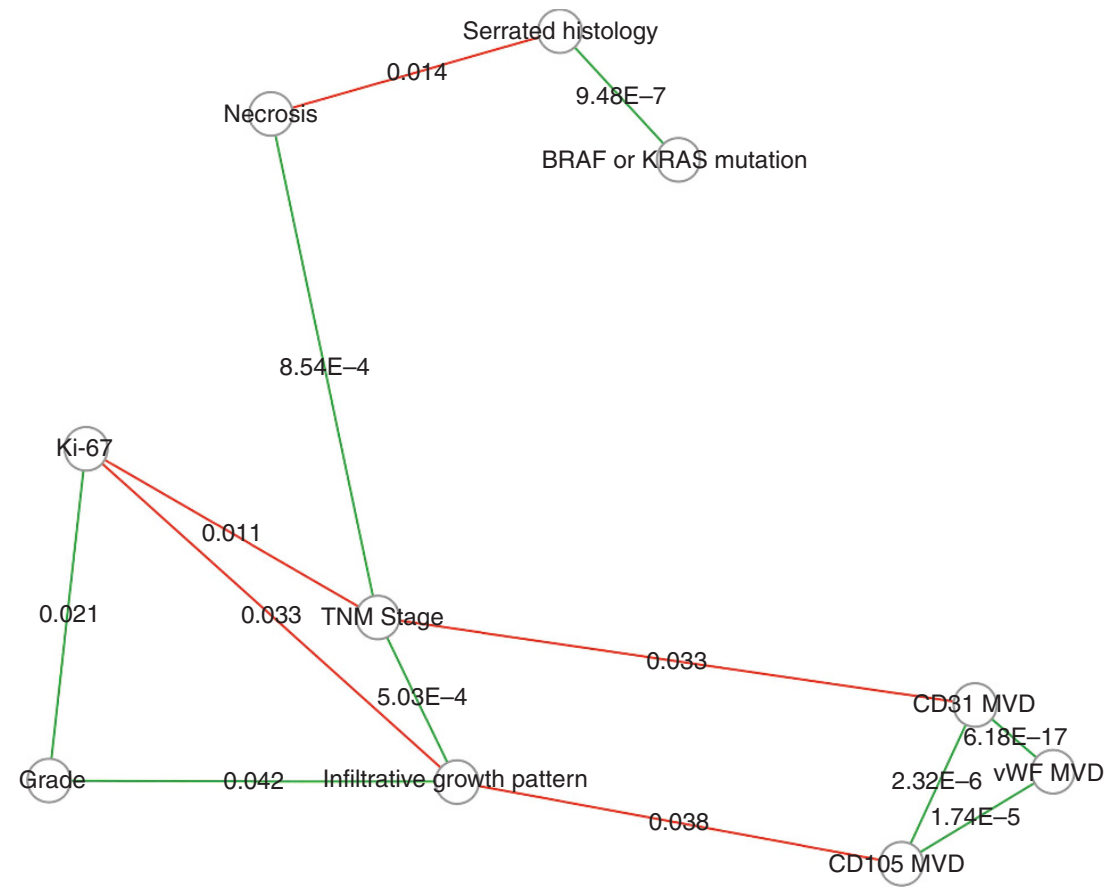

Figure 2. A graphical presentation of the interrelationships between tumour necrosis, biological properties of the tumour and clinicopathological variables. Individual variables are represented by nodes and their associations are represented by the connecting lines (edges). Only the associations with $P<0.05$ are shown, and the edge length illustrates the significance of the association. Green edges indicate a positive correlation and red edges indicate a negative correlation. The visualisation was created with Cytoscape software platform (Shannon et al, 2003) utilising the Prefuse force directed algorithm weighted by the statistical significances of the correlations between individual variables.

$\mathrm{MVD}=$ microvascular density; $\mathrm{TNM}=$ tumour, node and metastasis.

\section{Table 1. Correlations between tumour necrosis percentage, microvascular density and proliferation rate}

\begin{tabular}{|l|c|c|c|c|}
\hline & CD31 MVD & vWF MVD & CD105 MVD & Ki-67 \\
\hline Tumour necrosis & $0.020(P=0.818)$ & $0.004(P=0.961)$ & $-0.039(P=0.654)$ & $-0.131(P=0.126)$ \\
\hline CD31 MVD & 1 & $0.623(P=6.18 \mathrm{E}-17)$ & $0.383(P=2.32 \mathrm{E}-6)$ & $0.076(P=0.370)$ \\
\hline VWF MVD & $0.623(P=6.18 \mathrm{E}-17)$ & 1 & $0.351(P=1.74 \mathrm{E}-5)$ & $-0.051(P=0.546)$ \\
\hline CD105 MVD & $0.383(P=2.32 \mathrm{E}-6)$ & $0.351(P=1.74 \mathrm{E}-5)$ & 1 & $0.047(P=0.582)$ \\
\hline Abbreviations: MVD $=$ microvascular density; VWF $=$ von Willebrand factor. Numbers indicate Pearson's correlation coefficients for logarithmically transformed variables. \\
\hline
\end{tabular}

Determinants of tumour necrosis. Tumour necrosis (Figure 1) was present in 141 of 147 (95.9\%) cases. The median of tumour necrosis percentage was $10.0 \%$ (range 0-90.0\%; Supplementary Table S1). We first evaluated the network of determinants of tumour necrosis in CRC (Figure 2), and the associations between tumour necrosis percentage and clinicopathological characteristics are presented in Supplementary Table S3 and the associations between tumour necrosis, MVD and proliferation in Table 1.

Tumour necrosis was more extensive in higher stage tumours $(P=8.5 \mathrm{E}-4$; Supplementary Table $\mathrm{S} 3)$ and especially in those with higher $\mathrm{T}$ class $(P=4.0 \mathrm{E}-6)$. In SACs, tumour necrosis percentage was lower than in CCs (median $5.0 \%$ vs $11.0 \%$; $P=0.014$ ). Patient age, sex, tumour location, WHO grade, infiltrative tumour growth pattern or MMR screening status did not show significant associations with tumour necrosis. The patients who received preoperative RT/CRT did not have significantly different tumour necrosis percentage relative to those who did not receive preoperative RT/CRT. Tumours with oncogenic mutations in KRAS or BRAF genes had some tendency towards lesser amount of tumour necrosis (CRC with KRAS or BRAF mutation $v s$ wt KRAS and BRAF, $P=0.172$ ). The different markers for MVD showed notable high intercorrelations but did not associate with tumour necrosis. Tumours with extensive necrosis showed a tendency towards lower proliferation rate $(\mathrm{Ki}$ -
67; $P=0.126)$. Preoperative RT/CRT associated with increased CD105 MVD $(P=0.021)$ but did not associate with other MVD markers or the proliferation rate (Supplementary Table S4).

Finally, to address, whether proliferation and MVD would be different in areas around necrosis relative to non-necrotic areas in the same tumours, CD31 and Ki-67 immunohistochemistry was conducted on whole-tissue specimens of 30 patients with $>10 \%$ necrosis. The results indicated that there were high correlations between Ki-67 percentage ( $r=0.941)$, as well as CD31 MVD $(r=0.893)$ in areas around and distant from necrosis (Supplementary Figure S1), suggesting that the proliferation rate and MVD in perinecrotic areas correspond with those of the non-necrotic parts of the same tumour.

Survival analysis. To enlighten the prognostic significance of tumour necrosis, a 60-month survival (DFS, CSS and OS) analysis was carried out. The follow-up and event data is presented in Supplementary Table S5.

First, the discriminatory capacity of tumour necrosis was studied with ROC analysis, which indicated that tumour necrosis associated with worse DFS, with an optimal cutoff point of 7-10\% for tumour necrosis percentage in discriminating the survivors from nonsurvivors (Figure 3A). We decided to apply 10\% because it was considered to be more easily reproducible. For CD31 MVD, 
A

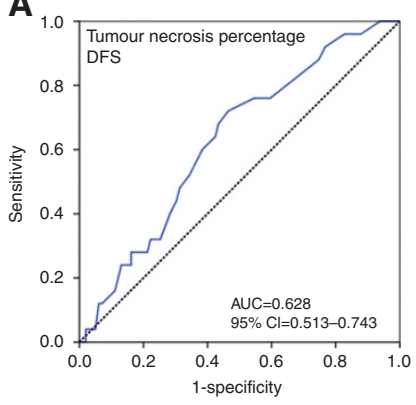

E

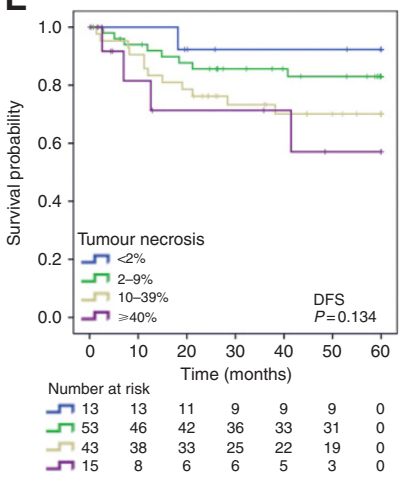

I

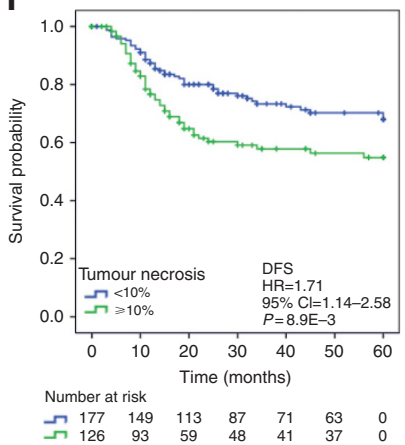

B

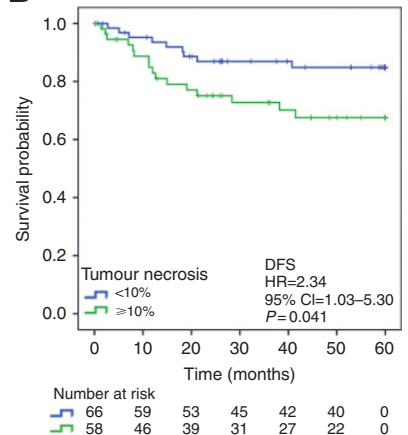

$\mathbf{F}$

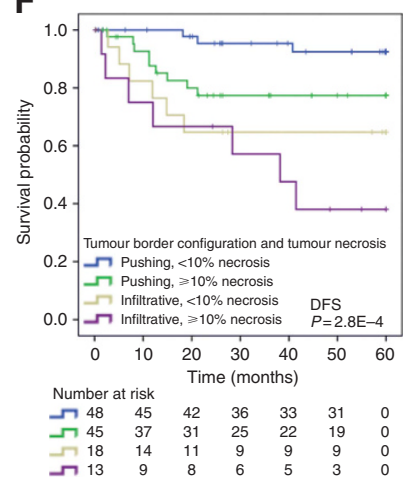

J

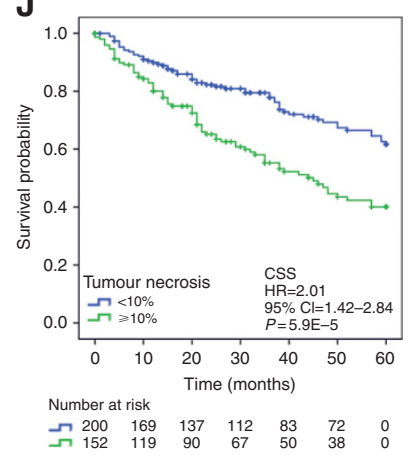

C

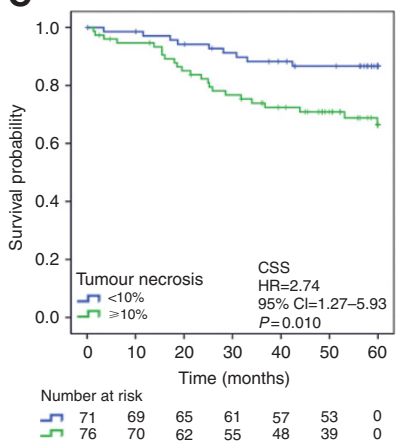

G

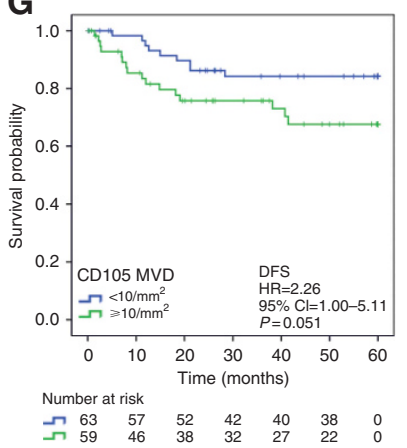

K

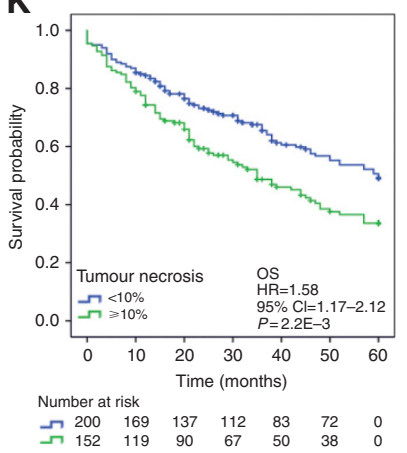

D

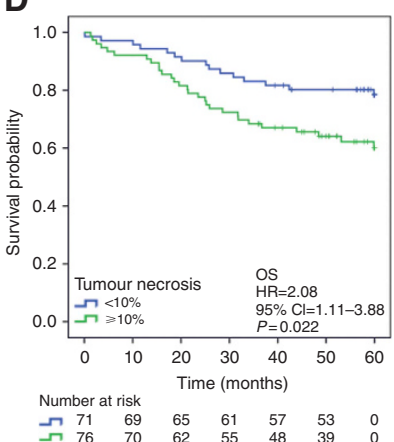

H

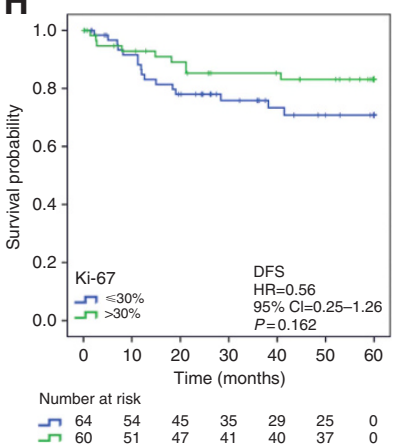

$\mathbf{L}$

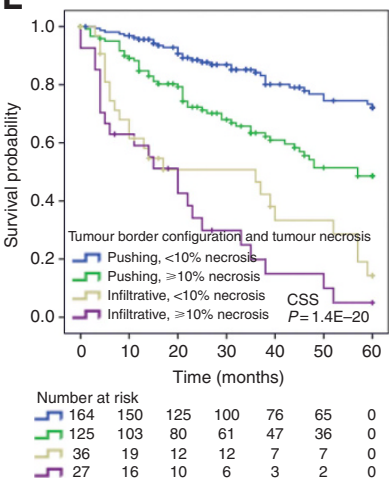

Figure 3. Survival analyses. (A-H) Study cohort. (A) Receiver operating characteristics (ROC) analysis for tumour necrosis in discriminating survivors from nonsurvivors. (B) Tumour necrosis and DFS. (C) Tumour necrosis and CSS. (D) Tumour necrosis and OS. (E) Tumour necrosis as a four-tiered variable and DFS. (F) Tumour necrosis, infiltrative growth pattern and DFS. (G) CD105 microvascular density (MVD) and DFS. (H) Proliferation rate and DFS. (I-L) Validation cohort. (I) Tumour necrosis and DFS. (J) Tumour necrosis and CSS. (K) Tumour necrosis and OS. (L) Tumour necrosis, infiltrative growth pattern and CSS.

vWF MVD, CD105 MVD and Ki-67 similarly defined cutoff points were $45,20,10 \mathrm{~mm}^{-2}$ and $30 \%$ respectively (Supplementary Table S6).

The two-tiered classification of tumour necrosis percentage $(<10 \% v s \geqslant 10 \%)$ had a strong association with DFS (Figure 3B, Supplementary Table S7), CSS (Figure 3C), and OS (Figure 3D). The adverse effect of tumour necrosis on survival was directly related to its extent (Figure 3E). Infiltrative tumours, regardless of the amount of necrosis, had worse prognosis comparing with expanding tumours (Figure 3F). The prognostic effect of tumour necrosis appeared to be parallel in SACs and CCs (data not shown). Of the studied immunohistochemical markers for MVD, high CD105 MVD associated with a tendency towards worse DFS $(P=0.054$, Figure $3 \mathrm{G})$. High proliferation rate showed a tendency towards better DFS $(P=0.170$, Figure $3 \mathrm{H})$.

Cox regression model indicated that high tumour necrosis percentage, infiltrative growth pattern and high CD105 MVD were indicators of worse DFS independent of tumour stage and other clinicopathological variables (Table 2). However, the associations between extensive tumour necrosis and worse CSS and OS were not independent of tumour stage (Table 2).

Validation cohort. To validate the prognostic significance of tumour necrosis, tumour necrosis percentage was evaluated in an independent cohort of 418 CRC patients. Patients' characteristics and correlations between tumour necrosis and clinicopathological features are summarised in Supplementary Data (Supplementary Tables S1 and S8). The follow-up and event data are presented in Supplementary Table S5.

Kaplan-Meier curves visualised that patients with extensive tumour necrosis $(\geqslant 10 \%)$ had significantly worse DFS (Figure 3I), CSS (Figure 3J) and OS (Figure 3K) than those with lesser amounts $(<10 \%)$ of necrosis. As in the study cohort, infiltrative tumours, regardless of the amount of necrosis, had worse prognosis comparing with expanding tumours (Figure 3L). Cox regression model indicated that the associations between high tumour 
Table 2. Cox regression model for the independent prognostic significance of necrosis in the study cohort

\begin{tabular}{|c|c|c|c|c|c|c|c|c|c|}
\hline & \multicolumn{3}{|c|}{ DFS } & \multicolumn{3}{|c|}{ CSS } & \multicolumn{3}{|c|}{ OS } \\
\hline Age ( $<65$ vs $\geqslant 65$ years $)$ & 3.69 & $1.25-10.9$ & 0.018 & 2.34 & $1.00-5.45$ & 0.049 & 2.39 & $1.18-4.81$ & 0.015 \\
\hline Nodal metastases (NO vs N1-N2) & 14.3 & $4.09-49.9$ & $3.10 \mathrm{E}-5$ & 3.97 & $1.45-10.9$ & $7.3 E-3$ & 2.96 & $1.51-5.81$ & $1.6 E-3$ \\
\hline Distant metastases (M0 vs M1) & - & - & - & 5.65 & $2.18-14.7$ & $3.9 E-4$ & 3.33 & $1.55-7.16$ & $2.1 E-3$ \\
\hline Preoperative RT/CRT (No vs Yes) & 0.66 & $0.19-2.34$ & 0.525 & 0.25 & $0.05-1.16$ & 0.077 & 0.49 & $0.17-1.40$ & 0.185 \\
\hline Serrated histology (No vs Yes) & - & - & - & - & - & - & - & - & - \\
\hline Infiltrative tumour border (No vs Yes) & 4.35 & $1.56-12.2$ & $5.1 \mathrm{E}-3$ & - & - & - & - & - & - \\
\hline $\begin{array}{l}\text { Lymphatic or blood vessel invasion } \\
\text { (No vs Yes) }\end{array}$ & - & - & - & 3.03 & $1.06-8.70$ & 0.039 & - & - & - \\
\hline
\end{tabular}

Table 3. Cox regression model for the independent prognostic significance of necrosis in the validation cohort

\begin{tabular}{|c|c|c|c|c|c|c|c|c|c|}
\hline & \multicolumn{3}{|c|}{ DFS } & \multicolumn{3}{|c|}{ CSS } & \multicolumn{3}{|c|}{ OS } \\
\hline & HR & $95 \% \mathrm{Cl}$ & $P$-value & HR & $95 \% \mathrm{Cl}$ & $P$-value & HR & $95 \% \mathrm{Cl}$ & $P$-value \\
\hline Age $(<65$ vs $\geqslant 65)$ & - & - & - & - & - & - & 1.87 & $1.34-2.61$ & $2.6 \mathrm{E}-4$ \\
\hline Tumour invasion (T1-T2 vs T3-T4) & 1.19 & $0.66-2.15$ & 0.559 & 1.30 & $0.71-2.38$ & 0.396 & 0.74 & $0.47-1.15$ & 0.181 \\
\hline Nodal metastases (N0 vs N1-N2) & 1.25 & $0.77-2.02$ & 0.367 & 1.88 & $1.22-2.88$ & $4.1 E-3$ & 1.44 & $1.00-2.06$ & 0.050 \\
\hline Distant metastases (M0 vs M1) & - & - & - & 4.95 & $3.16-7.73$ & $2.7 \mathrm{E}-12$ & 3.36 & $2.20-5.14$ & $2.1 E-8$ \\
\hline WHO Grade (1-2 vs 3) & - & - & - & 1.82 & $1.20-2.76$ & $5.2 E-3$ & 1.51 & $1.04-2.19$ & 0.031 \\
\hline Tumour location (Colon vs Rectum) & 1.84 & $1.21-2.79$ & $4.4 \mathrm{E}-3$ & 1.78 & $1.22-2.59$ & $2.5 E-3$ & 1.49 & $1.09-2.04$ & 0.013 \\
\hline Serrated growth pattern (No vs Yes) & 0.97 & $0.44-2.14$ & 0.941 & 1.27 & $0.69-2.31$ & 0.442 & 1.32 & $0.81-2.14$ & 0.263 \\
\hline $\begin{array}{l}\text { Lymphatic or blood vessel invasion (No vs } \\
\text { Yes) }\end{array}$ & 1.76 & $1.07-2.90$ & 0.025 & 1.80 & $1.18-2.74$ & $6.2 E-3$ & 1.83 & $1.28-2.62$ & $9.8 E-4$ \\
\hline Infiltrative tumour border (No vs Yes) & 3.50 & $2.08-5.91$ & $2.6 E-6$ & 2.57 & $1.73-3.84$ & $3.6 E-6$ & 2.44 & $1.70-3.51$ & $1.5 E-6$ \\
\hline Tumour necrosis $(<10 \%$ vs $\geqslant 10 \%)$ & 1.60 & $1.05-2.46$ & 0.031 & 1.84 & $1.27-2.67$ & $1.3 E-3$ & 1.51 & $1.10-2.06$ & 0.010 \\
\hline \multicolumn{10}{|c|}{$\begin{array}{l}\text { Abbreviations: } \mathrm{Cl}=\text { confidence interval; } \mathrm{CSS}=\text { cancer-specific survival; DFS=disease-free survival; } \mathrm{HR}=\text { hazard ratio; OS =overall survival; RT/CRT=radiotherapy/chemoradiotherapy } \\
\mathrm{TNM}=\text { tumour, node and metastasis. All the models included TNM stage variables, serrated histology, and tumour necrosis. Other variables were selected utilising the stepwise forwarc }\end{array}$} \\
\hline
\end{tabular}

necrosis percentage and worse DFS, CSS and OS were independent of tumour stage and other clinicopathological variables (Table 3). Tumour necrosis was also independent marker of worse DFS, CSS and OS in the subgroup of stage II patients (Supplementary Table S9). The inclusion of infiltrative growth pattern in the regression models was observed to emphasise the prognostic significance of tumour necrosis (data not shown), indicating that combined analysis of these variables improves the discriminatory capacity relative to individual variables.

\section{DISCUSSION}

In this study, we evaluated the biological mechanisms underlying tumour necrosis in CRC and the correlation of tumour necrosis with disease outcome. A graphical presentation of the interrelationships between different variables was created to facilitate the conceptualisation of the network of factors involved (Figure 2). The results indicated that the extent of tumour necrosis associates with high tumour stage-especially with high $\mathrm{T}$ class-and inversely correlates with serrated histology, but does not significantly associate with proliferation rate, MVD, and KRAS or BRAF mutation. Tumour necrosis associated with adverse DFS, CSS and OS in two independent CRC cohorts $(n=147$ and $n=418)$ and the association between tumour necrosis and adverse DFS was independent of other clinicopathological factors in both cohorts.

Tumour necrosis is a common feature of solid tumours, thought to reflect intratumoural hypoxic environment due to rapid increase of tumour cell numbers outstripping the vascular supply (Swinson et al, 2002). A few studies have indicated high proliferation rate to associate with extensive tumour necrosis in some tumour types such as in nodular melanoma and renal cell carcinoma (Bachmann et al, 2008; Pichler et al, 2012), while also conflicting results exist in, for example, oesophageal carcinoma (Dutta et al, 2012) and renal cell carcinoma (Tollefson et al, 2007). Our observations indicate that tumour necrosis is not associated with the rate of tumour cell proliferation in CRC. In our data, tumours with high proliferation rate showed a weak tendency towards better survival (DFS: $P=0.162$ ), and also earlier reports support the association between high proliferation and better survival in CRC (Allegra, 
2003; Roxburgh et al, 2013), while in, for example, breast cancer (Luporsi et al, 2012) and renal cell carcinoma (Tollefson et al, 2007), high proliferation rate has been associated with worse prognosis. The reasons underlying this inconsistency between different cancers are not clear. However, current results suggest that for the prognosis of CRC patients, other factors such as tumour immunogenicity (Roxburgh et al, 2013) or tumour growth pattern (Koelzer and Lugli, 2014) are more important than proliferation rate.

Angiogenesis is known to be crucial requirement for the tumour growth, enabling the tumour cells capable of rapid proliferation to rapidly expand their population (Folkman, 1990). The assessment of MVD utilising different endothelial cell markers is the standard method for evaluating the tumour vasculature (Nico et al, 2008). We hypothesised that tumour necrosis could result from the inadequate blood vessel supply of oxygen into the tumours, which could be seen as low MVD in highly necrotic CRCs. However, our results do not support this hypothesis, since there were no association between tumour necrosis percentage and CD31, CD105 and vWF MVD.

Neovascularisation contributes to the invasion and metastasis formation (Folkman, 1990) and high MVD has been shown to correlate with aggressiveness and poor outcomes in many cancers (Takagi et al, 2005), including CRC (Des Guetz et al, 2006). Our results indicate that, specifically, high CD105 MVD is a stage independent marker for worse prognosis (DFS: $P=0.027$ ), which is in accordance with the finding of Li et al (2003). CD105 is a hypoxia-inducible protein, primarily expressed in the actively proliferating endothelial cells participating in neoangiogenesis in tumours (Minhajat et al, 2006; Nico et al, 2008). In the vessels of normal tissues, its expression is generally undetectable or weak (Minhajat et al, 2006; Nico et al, 2008), while CD31 and vWF are present in the vast majority of the blood vessels of normal and cancer tissue (Minhajat et al, 2006; Pusztaszeri et al, 2006).

A few studies have reported tumour necrosis to be less common in SACs comparing with CCs (Tuppurainen et al, 2005). SACs frequently harbour oncogenic mutations in either BRAF or KRAS (Mäkinen, 2007; Stefanius et al, 2011), the participants of MAPKERK pathway controlling, for example, cell proliferation, differentiation and growth. In our study, BRAF and KRAS mutations associated with a tendency towards higher proliferation rate $(P=0.091)$ and with a tendency towards lower tumour necrosis percentage $(P=0.172)$, while SACs had similar proliferation rate $(P=0.354)$ but significantly lower tumour necrosis percentage relative to CCs $(P=0.014)$. This suggests that KRAS and BRAF mutations may contribute to the proliferation rate of the tumours but other factors account for the scarcity of tumour necrosis in SAC.

Neoadjuvant CRT is known for its ability to modify the histological appearance of rectal cancer, while the short course preoperative RT induces only little or no visible effects. Usually, the tumour tissue is replaced by fibrous/fibroinflammatory reaction or mucin collections, and decreased frequency of tumour necrosis has been reported (Shia et al, 2004; O'Neil and Damjanov, 2009). In this study, there was no statistical significant association between preoperative RT/CRT and tumour necrosis percentage. In addition, the limited number of the patients with preoperative RT/CRT $(n=31)$ did not allow for sensible survival analyses for this subgroup in this study. Subsequently, it would be important to establish, whether tumour necrosis has prognostic value also in this patient subgroup.

We conducted survival analyses for two independent CRC cohorts $(n=147$ and $n=418)$ and established that tumour necrosis has prognostic value (Study cohort: DFS; Validation cohort: DFS, CSS and OS) independent of tumour stage and other biological or clinicopathological characteristics. This finding is in agreement with previous studies (Mulcahy et al, 1997; Gao et al, 2005; Pollheimer et al, 2010; Richards et al, 2012). Interestingly, the
$\mathrm{T}$ classification was not significant in any of the Cox regression models, which could be related to the close association between tumour necrosis and $\mathrm{T}$ classification. The advantage of our study is the validation of the clinical impact of tumour necrosis in an independent cohort, the range of study end points (DFS, CSS and OS), as well as the evaluation of the tumour necrosis percentage as a continuous variable, expanding the available statistical methodology (Zlobec et al, 2007). The ROC analysis indicated $7-10 \%$ as an optimal cutoff value for necrosis percentage for discriminating survivors from nonsurvivors (DFS presented in Figure 3A; CSS and OS yielded parallel results although data not shown), and this serves as a reference for further studies. It was also established that the adverse effect of necrosis on survival was directly related to its extent, highlighting the relevance of quantitative necrosis percentage evaluation.

The greatest limitation of the study was the retrospective nature of analysis in the validation cohort. Imaging technologies have improved and may have allowed for earlier detection of recurrences in the study cohort relative to the validation cohort. Moreover, the lymph node sampling was not, at the time of the operation of patients in the validation cohort, as accurate as required in current standards, likely underestimating the effect of the $\mathrm{N}$ classification in the Cox regression models. Indeed, the $\mathrm{N}$ classification did not show a statistically significant independent association with DFS in the validation cohort. However, both cohorts were reviewed and reclassified using TNM6, and several additional histological prognostic factors were evaluated, which strengthens the quality of the cohort. Of these markers, the limitation in the analysis of lymphatic and blood vessel invasion was that vascular capillary invasion and lymphatic invasion cannot always be reliably distinguished. Therefore, Cox regression models included the combination variable lymphatic or blood vessel invasion. All the material used in this study was from a single pathology unit, and all surgery had been performed at Oulu University Hospital. The fixation times for surgical samples had remained constant during the years, so that fixation time is unlikely to affect the histological or immunohistochemical analyses.

TNM staging is the main prognostic factor in CRC, but additional prognostic factors are needed to further classify, especially, stage IIIII patients (Puppa et al, 2010). Infiltrative tumour growth represents one of the highest-rated additional histological prognostic factors (Koelzer and Lugli, 2014), associated with a worse prognosis in CRC independent of tumour stage in a number of studies (Jass et al, 1996; Morikawa et al, 2012). Our results indicate that tumours with infiltrative and pushing border configuration harbour similar amounts of tumour necrosis, regardless of the aggressive tumour behaviour associated with the infiltrative tumour growth. Accordingly, it was established that tumour necrosis would, especially, facilitate the prognostic classification of the patients with a pushing tumour border and the prognostic significance of tumour necrosis was emphasised in the Cox regression models, when also infiltrative growth was included. Taken together, the results suggest that infiltrative tumour growth and extensive tumour necrosis represent relevant histomorphological prognostic indicators that complement each other.

In conclusion, tumour necrosis associates with high tumour stage-especially with $\mathrm{T}$ class-in CRC and inversely correlates with serrated histology, but does not significantly associate with proliferation rate, MVD and KRAS or BRAF mutation. Evaluation of the tumour necrosis percentage provides additional prognostic information in CRC.

\section{ACKNOWLEDGEMENTS}

We thank Ms Riitta Vuento for her invaluable assistance in the preparation of the study material. This work was supported by 
grants from Finnish Medical Foundation, Ida Montin Foundation, Orion Research Foundation, and Vatsatautien tutkimussäätiö.

\section{CONFLICT OF INTEREST}

The authors declare no conflict of interest.

\section{REFERENCES}

Allegra CJ (2003) Prognostic value of thymidylate synthase, Ki-67, and p53 in patients with Dukes' B and C colon cancer: A National Cancer InstituteNational Surgical Adjuvant Breast and Bowel Project Collaborative Study. J Clin Oncol 21: 241-250.

Bachmann IM, Ladstein RG, Straume O, Naumov GN, Akslen LA (2008) Tumor necrosis is associated with increased alphavbeta 3 integrin expression and poor prognosis in nodular cutaneous melanomas. $B M C$ Cancer 8: 362.

Center MM, Jemal A, Smith RA, Ward E (2009) Worldwide Variations in Colorectal Cancer. CA Cancer J Clin 59: 366-378.

Des Guetz G, Uzzan B, Nicolas P, Cucherat M, Morere J-F, Benamouzig R, Breau J-L, Perret G-Y (2006) Microvessel density and VEGF expression are prognostic factors in colorectal cancer. Meta-analysis of the literature. Br J Cancer 94: 1823-1832.

Dutta S, Going JJ, Crumley ABC, Mohammed Z, Orange C, Edwards J, Fullarton GM, Horgan PG, McMillan DC (2012) The relationship between tumour necrosis, tumour proliferation, local and systemic inflammation, microvessel density and survival in patients undergoing potentially curative resection of oesophageal adenocarcinoma. Br J Cancer 106: 702710 .

Folkman J (1990) What is the evidence that tumors are angiogenesis dependent? J Natl Cancer Inst 82: 4-6.

Frank I, Blute ML, Cheville JC, Lohse CM, Weaver AL, Zincke H (2002) An outcome prediction model for patients with clear cell renal cell carcinoma treated with radical nephrectomy based on tumor stage, size, grade and necrosis: the SSIGN score. J Urol 168: 2395-2400.

Gao JF, Arbman G, Wadhra TI, Zhang H, Sun XF (2005) Relationships of tumor inflammatory infiltration and necrosis with microsatellite instability in colorectal cancers. World J Gastroenterol 11: 2179-2183.

Hamilton SR, Bosman FT, Boffetta P, Ilyas M, Morreau H, Nakamura SI, Quirke P, Riboli E, Sobin LH (2010) Carcinoma of the colon and rectum. In WHO Classification of Tumours of the Digestive System, Bosman F, Carneiro F, Hruban R, Theise N (eds). IARC Press: Lyon, pp 134-146.

Jass JR, Ajioka Y, Allen JP, Chan YF, Cohen RJ, Nixon JM, Radojkovic M, Restall AP, Stables SR, Zwi LJ (1996) Assessment of invasive growth pattern and lymphocytic infiltration in colorectal cancer. Histopathology 28: $543-548$.

Kantola T, Klintrup K, Väyrynen JP, Vornanen J, Bloigu R, Karhu T, Herzig K-H, Näpänkangas J, Mäkelä J, Karttunen TJ, Tuomisto A, Mäkinen MJ (2012) Stage-dependent alterations of the serum cytokine pattern in colorectal carcinoma. Br J Cancer 107: 1729-1736.

Kantola T, Väyrynen JP, Klintrup K, Mäkelä J, Karppinen SM, Pihlajaniemi T, Autio-Harmainen H, Karttunen TJ, Mäkinen MJ, Tuomisto A (2014) Serum endostatin levels are elevated in colorectal cancer and correlate with invasion and systemic inflammatory markers. Br J Cancer 111: 1605-1613.

Koelzer VH, Lugli A (2014) The tumor border configuration of colorectal cancer as a histomorphological prognostic indicator. Front Oncol 4: 29.

Li C, Gardy R, Seon BK, Duff SE, Abdalla S, Renehan A, O'Dwyer ST, Haboubi N, Kumar S (2003) Both high intratumoral microvessel density determined using CD105 antibody and elevated plasma levels of CD105 in colorectal cancer patients correlate with poor prognosis. $\mathrm{Br} J$ Cancer $\mathbf{8 8}$ : 1424-1431.

Luporsi E, André F, Spyratos F, Martin P-M, Jacquemier J, Penault-Llorca F, Tubiana-Mathieu N, Sigal-Zafrani B, Arnould L, Gompel A, Egele C, Poulet B, Clough KB, Crouet H, Fourquet A, Lefranc J-P, Mathelin C, Rouyer N, Serin D, Spielmann M, Haugh M, Chenard M-P, Brain E, de Cremoux P, Bellocq J-P (2012) Ki-67: level of evidence and methodological considerations for its role in the clinical management of breast cancer: analytical and critical review. Breast Cancer Res Treat 132: 895-915.
McShane LM, Altman DG, Sauerbrei W, Taube SE, Gion M, Clark GM (2005) REporting recommendations for tumour MARKer prognostic studies (REMARK). Br J Cancer 93: 387-391.

Minhajat R, Mori D, Yamasaki F, Sugita Y, Satoh T, Tokunaga O (2006) Endoglin (CD105) expression in angiogenesis of colon cancer: analysis using tissue microarrays and comparison with other endothelial markers. Virchows Arch 448: 127-134.

Morikawa T, Kuchiba A, Qian ZR, Mino-Kenudson M, Hornick JL, Yamauchi M, Imamura Y, Liao X, Nishihara R, Meyerhardt JA, Fuchs CS, Ogino S (2012) Prognostic significance and molecular associations of tumor growth pattern in colorectal cancer. Ann Surg Oncol 19: 1944-1953.

Mulcahy HE, Toner M, Patchett SE, Daly L, O’Donoghue DP (1997) Identifying stage B colorectal cancer patients at high risk of tumor recurrence and death. Dis Colon Rectum 40: 326-331.

Mäkinen MJ (2007) Colorectal serrated adenocarcinoma. Histopathology 50: 131-150.

Nico B, Benagiano V, Mangieri D, Maruotti N, Vacca A, Ribatti D (2008) Evaluation of microvascular density in tumors: pro and contra. Histol Histopathol 23: 601-607.

O’Neil M, Damjanov I (2009) Histopathology of Colorectal Cancer after Neoadjuvant Chemoradiation Therapy. Open Pathol J 3: 91-98.

Pichler M, Hutterer GC, Chromecki TF, Jesche J, Kampel-Kettner K, Rehak P, Pummer K, Zigeuner R (2012) Histologic tumor necrosis is an independent prognostic indicator for clear cell and papillary renal cell carcinoma. Am J Clin Pathol 137: 283-289.

Pollheimer MJ, Kornprat P, Lindtner RA, Harbaum L, Schlemmer A, Rehak P, Langner C (2010) Tumor necrosis is a new promising prognostic factor in colorectal cancer. Hum Pathol 41: 1749-1757.

Puppa G, Sonzogni A, Colombari R, Pelosi G (2010) TNM Staging System of Colorectal Carcinoma A Critical Appraisal of Challenging Issues. Arch Pathol Lab Med 134: 837-852.

Pusztaszeri MP, Seelentag W, Bosman FT (2006) Immunohistochemical expression of endothelial markers CD31, CD34, von Willebrand factor, and Fli-1 in normal human tissues. J Histochem Cytochem 54: 385-395.

Richards CH, Roxburgh CS, Anderson JH, McKee RF, Foulis AK, Horgan PG, McMillan DC (2012) Prognostic value of tumour necrosis and host inflammatory responses in colorectal cancer. Br J Surg 99: 287-294.

Roxburgh CS, Richards CH, Macdonald AI, Powell AG, McGlynn LM, McMillan DC, Horgan PG, Edwards J, Shiels PG (2013) The in situ local immune response, tumour senescence and proliferation in colorectal cancer. Br J Cancer 109: 2207-2216.

Sajanti S, Sirniö P, Väyrynen JP, Tuomisto A, Klintrup K, Mäkelä J, Ristimäki A, Mäkinen MJ (2014) VE1 immunohistochemistry accurately detects BRAF V600E mutations in colorectal carcinoma and can be utilized in the detection of poorly differentiated colorectal serrated adenocarcinoma. Virchows Arch 464: 637-643.

Shannon P, Markiel A, Ozier O, Baliga NS, Wang JT, Ramage D, Amin N, Schwikowski B, Ideker T (2003) Cytoscape: a software environment for integrated models of biomolecular interaction networks. Genome Res 13: 2498-2504.

Shia J, Guillem JG, Moore HG, Tickoo SK, Qin J, Ruo L, Suriawinata A, Paty PB, Minsky BD, Weiser MR, Temple LK, Wong WD, Klimstra DS (2004) Patterns of morphologic alteration in residual rectal carcinoma following preoperative chemoradiation and their association with long-term outcome. Am J Surg Pathol 28: 215-223.

Sobin LH, Wittekind C (2002) TNM Classification of Malignant Tumours. Wiley-Liss: New York, NY, USA.

Stefanius K, Ylitalo L, Tuomisto A, Kuivila R, Kantola T, Sirniö P, Karttunen TJ, Mäkinen MJ (2011) Frequent mutations of KRAS in addition to BRAF in colorectal serrated adenocarcinoma. Histopathology 58: 679-692.

Swinson DEB, Jones JL, Richardson D, Cox G, Edwards JG, O’Byrne KJ (2002) Tumour necrosis is an independent prognostic marker in non-small cell lung cancer: correlation with biological variables. Lung Cancer 37: 235-240.

Takagi K, Takada T, Amano H (2005) A high peripheral microvessel density count correlates with a poor prognosis in pancreatic cancer. $J$ Gastroenterol 40: 402-408.

Tollefson MK, Thompson RH, Sheinin Y, Lohse CM, Cheville JC, Leibovich BC, Kwon ED (2007) Ki-67 and coagulative tumor necrosis are independent predictors of poor outcome for patients with clear cell renal cell carcinoma and not surrogates for each other. Cancer 110: 783-790.

Tuppurainen K, Makinen JM, Junttila O, Liakka A, Kyllonen AP, Tuominen H, Karttunen TJ, Makinen MJ (2005) Morphology and microsatellite 
instability in sporadic serrated and non-serrated colorectal cancer. J Pathol 207: 285-294.

Väyrynen JP, Kantola T, Väyrynen SA, Klintrup K, Bloigu R, Karhu T, Mäkelä J, Herzig K-H, Karttunen TJ, Tuomisto A, Mäkinen MJ (2016) The relationships between serum cytokine levels and tumor infiltrating immune cells and their clinical significance in colorectal cancer. Int $J$ Cancer 139: 112-121.

Väyrynen JP, Sajanti SA, Klintrup K, Mäkelä J, Herzig K-H, Karttunen TJ, Tuomisto A, Mäkinen MJ (2014) Characteristics and significance of colorectal cancer associated lymphoid reaction. Int J Cancer 134: $2126-2135$.

Väyrynen JP, Tuomisto A, Klintrup K, Mäkelä J, Karttunen TJ, Mäkinen MJ (2013) Detailed analysis of inflammatory cell infiltration in colorectal cancer. Br J Cancer 109: 1839-1847.

Väyrynen JP, Vornanen J, Tervahartiala T, Sorsa T, Bloigu R, Salo T, Tuomisto A, Mäkinen MJ (2012) Serum MMP-8 levels increase in colorectal cancer and correlate with disease course and inflammatory properties of primary tumors. Int J Cancer 131: E463-E474.

Weidner N, Semple JP, Welch WR, Folkman J (1991) Tumor angiogenesis and metastasis -correlation in invasive breast carcinoma. N Engl J Med 324: $1-8$.

Zlobec I, Steele R, Terracciano L, Jass JR, Lugli A (2007) Selecting immunohistochemical cut-off scores for novel biomarkers of progression and survival in colorectal cancer. J Clin Pathol 60: $1112-1116$.

This work is published under the standard license to publish agreement. After 12 months the work will become freely available and the license terms will switch to a Creative Commons AttributionNonCommercial-Share Alike 4.0 Unported License.

Supplementary Information accompanies this paper on British Journal of Cancer website (http://www.nature.com/bjc) 